University of Wollongong

Research Online

Faculty of Social Sciences - Papers (Archive) Faculty of Arts, Social Sciences \& Humanities

2013

Counterfactual thinking and anticipated emotions enhance performance in computer skills training

Amy Y.C Chan

University of Wollongong, amychan@uow.edu.au

Peter Caputi

University of Wollongong, pcaputi@uow.edu.au

Rohan Jayasuriya

University Of New South Wales, r.jayasuriya@unsw.edu.au

J L. Browne

University of Wollongong

Follow this and additional works at: https://ro.uow.edu.au/sspapers

Part of the Education Commons, and the Social and Behavioral Sciences Commons

Research Online is the open access institutional repository for the University of Wollongong. For further information contact the UOW Library: research-pubs@uow.edu.au 


\title{
Counterfactual thinking and anticipated emotions enhance performance in computer skills training
}

\author{
Abstract \\ The present study examined the relationship between novice learners' counterfactual thinking (i.e. \\ generating what if and if only thoughts) about their initial training experience with a computer application \\ and subsequent improvement in task performance. The role of anticipated emotions towards goal \\ attainment in task performance was also assessed. Undergraduate students $(N=42)$ with minimal \\ experience in using computer spreadsheets underwent basic training in using Microsoft Excel. All \\ participants were assessed on their anticipated positive and negative emotions regarding goal attainment \\ at the outset. After completing their first task, participants allocated to a counterfactual condition \\ received instructions to generate counterfactual thoughts regarding their initial task performance, \\ whereas participants in a control condition did not. The counterfactual group showed only marginally \\ greater improvement in task performance (measured by task completion time and accuracy) than the \\ control group. However, we also found that positive anticipated emotions were associated with \\ improvement in task performance but for the counterfactual group only. Our data have implications for \\ incorporating counterfactual thinking into information technology skills training to enhance learning \\ outcomes for novice learners.
}

\section{Keywords}

skills, computer, performance, enhance, emotions, anticipated, training, thinking, counterfactual

\section{Disciplines}

Education | Social and Behavioral Sciences

\section{Publication Details}

Chan, A. Y.C., Caputi, P., Jayasuriya, R. \& Browne, J. L. (2013). Counterfactual thinking and anticipated emotions enhance performance in computer skills training. Behaviour and Information Technology, 32 (4), 387-396. 
Running Head: Counterfactual Thinking and IT Skills Training

Counterfactual Thinking and Anticipated Emotions Enhance Performance in Computer Skills Training

Amy Y. C. Chan ${ }^{\mathrm{a}}$, Peter Caputi ${ }^{\mathrm{a}}$, Rohan Jayasuriya ${ }^{\mathrm{b}}$, and Jessica L. Browne ${ }^{\mathrm{a}}$

${ }^{\text {a }}$ School of Psychology, University of Wollongong, North Wollongong, NSW 2522,

Australia. (Email addresses: amychan@uow.edu.au, pcaputi@uow.edu.au, jlb96@uow.edu.au)

${ }^{\mathrm{b}}$ School of Public Health and Community Medicine , University of New South Wales, Sydney, NSW 2052, Australia (r.jayasuriya@unsw.edu.au).

Corresponding Author:

Dr. Amy Chan

School of Psychology

University of Wollongong

North Wollongong NSW 2522

Australia

Email: amychan@uow.edu.au

Phone: (+61 2) 4221-4468

Fax: (+61 2) 4221-4163 


\begin{abstract}
The present study examined the relationship between novice learners' counterfactual thinking (i.e., generating "what if” and "if only” thoughts) about their initial training experience with a computer application and subsequent improvement in task performance. The role of anticipated emotions toward goal attainment in task performance was also assessed. Undergraduate students $(N=42)$ with minimal experience in using computer spreadsheets underwent basic training in using Microsoft Excel. All participants were assessed on their anticipated positive and negative emotions regarding goal attainment at the outset. After completing their first task, participants allocated to a counterfactual condition received instructions to generate counterfactual thoughts regarding their initial task performance, whereas participants in a control condition did not. The counterfactual group showed only marginally greater improvement in task performance (measured by task completion time and accuracy) than the control group. However, we also found that positive anticipated emotions were associated with improvement in task performance, but for the counterfactual group only. Our data have implications for incorporating counterfactual thinking into IT skills training to enhance learning outcomes for novice learners.
\end{abstract}

Keywords: COUNTERFACTUAL THINKING, ANTICIPATED EMOTIONS, INFORMATION TECHNOLOGY TRAINING 
Counterfactual Thinking and Anticipated Emotions Enhance Performance in Computer Skills Training

\section{Introduction}

The rapid implementation of computerized information systems in diverse workplaces has resulted in training staff in application software. Increasingly more people are also taking part in computer and information technology (IT) skills training courses to meet the demands of modern everyday life. Such training may range from equipping the novice with basic computer skills, to educating experienced computer users on new software or advanced features of existing software. Different training methods have been reported in the literature to enhance IT skills acquisition. Early research concentrated on mastery training (asking learners to observe and model the behaviour required for successful task completion) (Gist et al. 1989) and guided exploration (Debrowski et al. 2001). More recently, methods to enhance general reflective thinking were used in error management training (encouraging learners to learn through making and reflecting on errors) (Keith and Frese 2005).

A technique that relies on our ability to reflect on past experience and to imagine what could have been different to produce possible alternative, counterfactual versions of outcome to reality, has been shown to facilitate human problem solving and decision making (Gakinsky and Kray 2004, Galinsky and Moskovitz 2000). Counterfactual thinking is conceptually akin to error management in that both techniques rely on the core human competence in reflecting on past experience. However, counterfactual thinking is distinct from error management in that the former further entails identifying alternative pathways to result in a different outcome. To date, this technique of counterfactual thinking has received little attention in improving task performance in IT training. The purpose of this study was to examine the effects of counterfactual thinking in improving novice learners’ task performance in learning to use a software application. 


\subsection{Counterfactual thinking}

Counterfactual thinking is characterised by thoughts of "what if” and "if only”. Such reconstruction of past events, to imagine alternative outcomes, is a natural and pervasive occurrence in many aspects of life (Roese and Olson 1995). Counterfactual thoughts are often classified according to their direction. Upward counterfactuals entail imagined alternatives better than actuality. For example, after their initial learning experience with a spreadsheet application, learners may ponder how their task performance could have been better: "If only I had paid more attention to the instructions, I would have understood better what I was supposed to do.” In contrast, downward counterfactuals are imagined alternatives worse than actuality. For example, the novice learner may ponder instead, "If only I had not been given an instruction sheet that stated the task clearly, I would have no idea where to even get started.”

The notion that counterfactuals can prepare individuals better in future is particularly relevant to the training context. Early research has focussed on demonstrating the utility of upward counterfactual thinking in enhancing intentions to engage in success-facilitating behaviour in future (e.g., Harris et al. 1996, Mandel and Lehman 1996, Markman et al. 1993) and improving actual task performance (e.g., Roese and Olson 1993; Roese 1994). However, the major premise of the present study is built upon more recent findings in this field of research, which suggest that it is the process of counterfactual thinking, rather than the specific counterfactual direction or content per se, that is beneficial to problem solving and decision making (Galinsky and Moskovitz 2000). By considering the logical relationships among events to identify alternative pathways to reality, the process of counterfactual thinking may trigger a broader mind-set that is conducive to seeking different ways to achieve a desirable goal. This should, in turn, help one overcome any tendency to become fixated in a single solution, and fail to realise that there may be better ways to 
achieve a good outcome (Galinsky and Moskovitz 2000, Galinsky and Kray 2004; Kray and Galinsky 2003). Furthermore, this mind-set occurs regardless of whether upward or downward counterfactuals are called upon. This counterfactual mind-set has been shown to facilitate better performance in a divergent thinking task that required overcoming fixations on conventional ways of thinking, and identifying creative solutions to a problem (Galinsky and Moskovitz 2000, Experiment 1); and in decision making tasks that required gathering and scrutinising information from diverse sources rather than focussing on a single tentative solution (Galinsky and Moskovitz, 2000, Experiment 3, Kray and Galinsky 2003). Kray et al. (2006) demonstrated that participants induced to be in a counterfactual mind-set performed better than control participants in an analytical reasoning task that required them to understand and apply given rules, and to analyse problems in order to identify how concepts were related and to draw appropriate conclusions (Experiment 2); and also in a remote associations test that entailed identifying a unique logical association among sets of distinct words (Experiment 5). These findings have since been replicated in different analytical problem solving contexts (Markman et al. 2007).

When learning an IT skill such as a spreadsheet application, the learner has to become familiar with the general layout of the application environment and the relevant tools available. Importantly, as the learner gains experience with using equations and functions to calculate, format, and carry out other complex tasks, they need to increasingly analyse the logical relationship between the procedure used and the resulting display in order to complete tasks efficiently. In view of the nature of counterfactual thinking and the accumulating evidence of its utility in facilitating task performance, particularly those tasks that involve examining logical relationships and applying analytical skills, there are theoretical and empirical grounds to hypothesise that counterfactual thinking incorporated into training programs would facilitate learning outcomes in IT training. However, to date the potential 
role of counterfactual thinking in facilitating learning outcomes in IT training is largely unexplored.

\subsection{Anticipated emotions and IT training}

It has been suggested that anticipated affective reactions to performance or nonperformance of behaviour are important determinants of intentions (van der Plight and de Vries 1998), and that specifically, appraisal of future states (success and failure) evokes anticipatory emotions that result in self regulatory processes (Carver and Scheirer 1998). These self regulatory processes are of theoretical interest in explaining behaviour decision and performance. In a parallel area of research on goal directed behaviour, Baggozi and Dholakia (2006) showed that anticipated emotions have had a positive effect on intentions and decisions to act toward goal-directed behaviours. The potential relevance of anticipated emotions to IT training - and indeed most forms of performance-oriented training - lies in their focus on anticipating the success or failure to attain specific learning goals (Bagozzi and Dholakia 2006). By anticipating the negative emotions caused by one's choice of behaviour, the likelihood of that particular (maladaptive) behaviour actually occurring in the future can be decreased, as individuals tend to behave in ways to minimise their chances of experiencing regret (Page and Colby 2003). Thus, it follows that anticipating positive emotions caused by goal attainment may also increase intentions to behave in ways that are perceived to enhance success.

Anticipated emotions are conceptually compatible with counterfactual thinking, in that the anticipatory simulation and evaluation of events relevant to achieving or failing to achieve one’s goals involves a special type of forward-looking counterfactual thinking, termed prefactual thinking (Gleicher et al. 1995). This conceptual link is made explicitly in the model of goal-directed behaviour (MGB; Perugini and Bagozzi 2001), which posits that individuals' intentions to engage in goal-directed behaviour are influenced by their desire to 
perform the act, which is in turn influenced by a variety of factors that include positive and negative anticipated emotional appraisals of goal achievement and goal failure. Note however, that there is an important distinction between anticipated emotions and prefactual thinking: Whereas prefactual thinking (similar to counterfactual thinking) entails mentally simulating events and actions relevant to goal-directed behaviour, anticipated emotions are derived from appraising the emotional outcome of goal achievement versus failure, without necessarily involving the identification of specific pathways and actions that may result in goal achievement or failure.

The effects of positive and negative emotions on desires, intentions and/or actual goal-directed behaviour have been investigated in IT-oriented domains, but with mixed findings. For example, negative but not positive anticipated emotions were found to significantly predict an increase in intentions to participate in open source software user groups (Bagozzi and Dholakia 2006). However, both positive and negative anticipated emotions have been shown to correlate positively with outcomes (examination scores) when learning to use statistical software in a four-week training program (Leone et al. 2004). Importantly, Leone et al.’s findings indicated that high magnitudes of both positive and negative anticipated emotions significantly predicted successful goal attainment, even when the influence of studying and practising behaviours was statistically controlled for. The latter finding is of particular relevance to the present study, as the effect of anticipated emotions on task performance following counterfactual thinking has not been explored. If anticipated emotions were predictive of goal attainment in computer skills training (Leone et al. 2004), and if counterfactual thinking were useful in augmenting task performance, then it follows that counterfactual thinking may be a particularly effective intervention to augment training performance for trainees who have high levels of anticipated emotions toward goal attainment. 


\subsection{Overview of the present study}

The aims of the present study were twofold. Our primary aim was to determine the effect of counterfactual thinking on task performance in a sample of undergraduate students who had minimal experience in using a computer spreadsheet software. Our secondary aim was to examine the effect of anticipated emotions on training outcomes in learning to use a computer spreadsheet software, in students who generate counterfactual thoughts (and those who do not) about their task performance during training.

Three hypotheses were examined in this study. Our first hypothesis was that learners who were encouraged to think counterfactually about their task performance would subsequently show greater improvement in task performance than learners who were not encouraged to think counterfactually. This hypothesis was examined via instructing one group of learners to think counterfactually about their initial task performance, while depriving a control group of learners of the opportunity to naturally generate counterfactual thoughts about their performance (see Roese and Olson 1995).

Our second hypothesis was concerned with the theoretical and conceptual links among anticipated emotions, counterfactual thinking and performance (Perugini and Bagozzi 2001, Leonie et al. 2004). We hypothesised that for learners who are encouraged to think counterfactually, those with higher levels of anticipated emotions (both positive and negative) toward successfully learning the spreadsheet software will show greater improvement in task performance.

It is possible that anticipated emotions were found in previous studies to facilitate goal attainment because software users had naturally occurring (but not measured) counterfactual thoughts about their training experience with using the software. Hence, our third hypothesis was that for learners who are not given the opportunity to think 
counterfactually (i.e., the control group), the magnitude of their positive and negative anticipated emotions will be unrelated to improvements in their task performance.

\section{Method}

\subsection{Participants}

The sample consisted of 42 undergraduate psychology students between 17 and 39 years (13 males, 29 females; mean age $=20.30$ years, $S D=4.04$ years $)$ at the University of Wollongong who participated voluntarily for subject credit. All participants were novices in using Microsoft-Excel ${ }^{\circledR}$, as established by their verbal self-report at the outset of their participation, as well as observation of their task performance via a screen-capturing software (see Materials) ${ }^{1}$.

\subsection{Materials}

\subsubsection{Camtasia}

The screen-capturing software Camtasia was used to generate standardised demonstrations of basic procedures and functions in Excel. Camtasia was also used to record participants' task performance, to enable the researchers to monitor that participants were in fact using relevant procedures for task completion, rather than using advanced functions or typing answers directly into various cells in Excel (see Footnote 1).

\subsubsection{Pre-recorded short demonstrations}

We generated two pre-recorded short demonstrations of basic procedures and functions in Excel with Camtasia to be used in the training phase. The first demonstration was designed to provide participants with an initial experience using Excel. It covered basic procedures used in Excel, which included opening the Excel application, entering data, changing cell width, using the =, +, -, *, and / keys and clicking on appropriate cells in the spreadsheet to carry out simple arithmetic operations (addition, subtraction, multiplication, division), as well as copying and pasting information. The second demonstration was 
designed to allow participants to experience completing a set of simple calculations in context. It showed a worked example on calculating gross and net profits for a fictitious small café business. It involved additional practice on data entry, copying and pasting information, as well as an introduction to using basic functions ("SUM” and "AVERAGE”) to calculate the sum as well as arithmetic mean of data from selected cells. The demonstrations were shown via a computer data projector. These two demonstrations were 2 mins 35 secs and 10 mins 39 secs in duration, respectively.

\subsubsection{Experimental tasks}

We developed two experimental tasks to assess participants’ ability to independently apply the procedures and functions in two novel contexts. One task was framed in the context of a property investor using Excel to calculate his earnings from buying and selling three investment properties. The other task was set in the context of a woman who used Excel to calculate her earnings from selling three types of hand-made chocolate Easter eggs.

For each experimental task, we generated an Excel workbook with two worksheets: the first worksheet contained all relevant headings and basic data entry already completed for the experimental task at hand (see Figure 1 for the setup of the Property task); the second one contained the full data sets and completed solutions for the two demonstrations ${ }^{2}$. Each experimental task was accompanied by an instruction sheet that provided the context and a set of required calculations specified in 10 steps. The steps entailed basic arithmetic operations, copying and pasting, and using the SUM and AVERAGE functions. The instructions for both experimental tasks were comparable in wording and length (Property Task: 198 words/1170 characters; Chocolate Task: 191 words/1188 characters). To control for differences in participants’ prior knowledge in economics, each instruction sheet also included basic directions for completing each step within the task without specifying the Excel commands required (e.g., "Calculate No. of Years Held for the Suburb A property by 
subtracting year purchased from year sold” - see Appendix A for full instructions for each experimental task and number of answers required for each step in each task). The procedures and operations involved in the demonstrations and experimental tasks were similar in complexity to those in other studies on computer spreadsheet training (e.g., Yi and Davis 2003).

\subsubsection{Counterfactual thinking task}

This task was a paper-and-pencil task completed on a one-page worksheet. The top half of the worksheet contained instructions and blank space for participants to write down a full description of their performance on the Excel task just attempted. The description provided the context for counterfactual thinking. The bottom half of the worksheet contained instructions for participants to record as many "what if" and "if only" thoughts as possible on how things could have been different about their performance on the task just completed.

\subsubsection{Torrance Test of Creativity}

In addition to materials specifically developed for the present study, we utilized one figural subtest of the Torrance Tests of Creativity (Torrance et al. 1990) as a cognitively engaging filler task. This subtest requires the participant to add lines to some incomplete figures to sketch novel objects or figures, and to make up a title for each drawing. This task was administered to participants in the control condition in lieu of the counterfactual thinking task (see Design and procedure), and was intended to minimise opportunities for participants in the control condition to generate counterfactual thoughts spontaneously.

\subsubsection{Anticipated emotions measure}

This 17-item measure of anticipated emotions (Perugini and Bagozzi 2001) requires participants to indicate the extent to which they would feel certain positive and negative emotions while contemplating doing a task. Specifically, they are asked "If I succeed in achieving GOAL X (a self determined goal relating to the task), I will feel...[excited, 
delighted, happy, glad, satisfied, proud, self-assured]”. They are also asked, "If I do not succeed in achieving GOAL X, I will feel...[angry, frustrated, guilty, ashamed, sad, disappointed, depressed, worried, uncomfortable, fearful]”. Each emotion is rated using an 11-point response scale where $0=$ "not at all”, and $10=$ "very much”.

[Insert Figure 1 about here]

\subsection{Design and procedure}

Participants took part in a single one-hour experimental session in a computer teaching laboratory with up to seven other participants. Each participant was seated in front of a personal computer with Excel and Camtasia installed, with the experimenter guiding participants through the pre-recorded demonstrations shown via a computer data projector screen. Experimental sessions were alternately assigned to either a counterfactual condition or control condition, and allocation of participants into these conditions was randomly determined via their voluntary sign-up for participation at various available session times ${ }^{3}$.

\subsubsection{Training phase}

After asking participants to confirm that they had not previously used Excel, the experimenter told participants that they would learn some basic Excel skills to enter data and carry out calculations. Participants were then instructed to complete the anticipated emotions measure. Afterwards, the following instructions were given:

“As we go through each example, I will show you a pre-recorded demonstration on the large screen. I want you to follow the demonstration to create your own copy of the example on your computer. This is important because it will help you get a good idea of how to do things yourself later on. So please make sure you pay close attention to the demonstrations."

Participants then watched the first demonstration task on screen and followed the procedure to generate their own spreadsheet. The experimenter paused the demonstration as 
necessary to address questions and to ensure that all participants had sufficient time to reproduce the content of the demonstration task. The same procedure was used in presenting the second demonstration task. The training phase was immediately followed by the test phase.

\subsubsection{Test phase}

The experimenter informed participants that their next task was to attempt a problem on their own, using their newly learned skills from the demonstrations. Next, participants received the instruction sheet for their first experimental task (thereafter referred to as “Task 1”), and were instructed to attempt it on their own. Ten minutes were allowed for this task, and participants were instructed to perform the task efficiently. Participants were told that their Excel workbook for the current task included all the data and procedures completed in the demonstrations, which they could consult if necessary by clicking on the flap for the second worksheet. Participants then followed the experimenter's prompts to open the Camtasia program to begin recording their activities on the computer, and opened the experimental task file in Excel to work through the steps required. At the end of the 10minute period, participants were asked to save their work and to stop the Camtasia recording.

At this point, participants were issued either the counterfactual thinking task (counterfactual thinking condition) or the figural subtest of the Torrance Tests of Creativity (control condition). Participants in both conditions were given 10 minutes to complete their task. Subsequently, the experimenter instructed participants to complete an unexpected second experimental task ${ }^{4}$ (thereafter referred to as “Task 2”) independently, and to record their actions via Camtasia. The instructions and procedure were the same as those in Task 1. Participants were again instructed to complete the task efficiently.

The order in which participants attempted the Property and Chocolate experimental tasks was counterbalanced across participants. The key dependent variables in this study were 
the total number of correct answers provided for each experimental task (max. $=19$ for each experimental task) and the total amount of time spent completing each experimental task $(\max .=600$ secs $)$.

3. Results

\subsection{Counterfactual thinking task}

Participants in the counterfactual condition recorded a total of 69 thoughts in response to the solicitation of counterfactual thoughts about how their task performance could have been different. Based on Tsiro and Mittal’s (2000) criteria for classifying responses as "what if”/counterfactual thoughts, we counted responses "that alter reality, create hypothetical scenarios, or express an opinion as to what might have been had a different decision been made” (Tsiro and Mittal 2000, p.411). Two independent raters coded the responses according to these criteria. Interrater agreement was $87.18 \%$. Disagreements were resolved through discussion.

Sixty-three counterfactual thoughts were thus identified. All 21 participants in the counterfactual condition explicitly recorded at least one counterfactual thought $(M=3.05, S D$ $=1.50$; range $=1$ to 7 ). For example, "If I had not misinterpreted the instructions, I might have performed better.”; and “If the sheet wasn’t in as much detail in describing what they wanted me to do then it would have taken longer to do.” Further examples of the counterfactual thoughts generated by participants can be found in Appendix B.

The non-counterfactual thoughts were primarily participants' descriptions of their experience. Two examples of responses that were classified as non-counterfactual thoughts were, “[a] major problem with this task is the lag in the computer response time for the Excel program that is caused by the background screen recording program. The lag was distracting and caused me to [sic.] faulter in my actions and thought processes several times.” and "[t]he 
tasks sheet was slightly confusing with the subtraction questions. I may have done something wrong there.”

\subsection{Experimental task performance}

A preliminary analysis indicated that the task completion time for the property task was longer than that for the chocolate task. Averaged across different task orders and experimental conditions, participants' completion time was significantly longer for the property task $(M=514 \mathrm{sec}, S D=106 \mathrm{sec})$ than for the chocolate task $(M=436 \mathrm{sec}, S D=118$ sec), $t(41)=3.14, p<.01, \eta^{2}=.19$. However, participants' accuracy did not differ between the two tasks [property task: $M=16.70, S D=3.50$; chocolate task: $M=16.00, S D=4.10$; $\left.t(41)=0.81, p>.4, \eta^{2}=.02\right]$

To safeguard against any speed-accuracy trade off in participants' task performance, we adapted Roese’s (1994, Experiment 3) scoring procedure to generate a performance score for each participant in each experimental task. The score was based on the total number of accurately completed subtasks divided by the time required to complete them ${ }^{5}$. Figure 2 shows the mean performance scores for the two experimental conditions in the experimental tasks.

\section{[Insert Figure 2 about here]}

\subsubsection{Counterfactual thinking and task performance}

Although there was improvement in performance from Task 1 to Task 2 in both conditions, it appeared that change in performance was greater in the counterfactual condition (see Figure 2). However, a mixed design analysis of variance (ANOVA) with experimental task as the repeated measure yielded a non-significant interaction effect for experimental task by condition, $F(1,40)=2.34, p=.13, \eta^{2}=.06$. This finding did not support our first hypothesis that counterfactual thinking would enhance novice Excel users' task performance during training. 


\subsubsection{Anticipated emotions and task performance}

Our next set of analyses examined the role of positive and negative anticipated emotions in task performance. We found no difference in mean total positive emotion scores between the counterfactual condition $(M=53.76, S D=11.24)$ and the control condition $(M=$ 53.95, $S D=9.11), t(40)=.06, S E=3.16, p=.95, \eta^{2}<.0001$. There was no difference in mean total negative emotion scores between the counterfactual condition $(M=47.00, S D=$ 20.73) and the control condition $(M=41.38, S D=19.57)$ either, $t(40)=.90, S E=6.22, p=$ $.37, \eta^{2}=.02$. Thus any subsequent group-based difference observed in the relationship between anticipated emotions and task performance cannot be attributed to a priori group differences in anticipated emotions between the counterfactual and control conditions.

General linear modelling (GLM) was used to examine the relationship betweeneffect of anticipated emotions, condition and their interaction on task performance. Residual gain scores were used as a measure of change in performance. When considered together, only positive anticipated emotions predicted change in performance $[F(1, \underline{36} 39)=\underline{9.08} 10.34, p=$ $\left..00 \underline{5} 3, \eta^{2}=.2 \underline{01}\right]$. There was no significant interaction between either positive or negative anticipated emotions and change in performance. This finding suggests that improvement in performance is associated with positive anticipated emotions and not negative anticipated emotions. Although there was no statistically significant interaction between anticipated emotion and condition, we conducted Sseparate GLMs conductedfor counterfactual thinking and control conditions to further explore this relationship. The results showed that for participants in the counterfactual condition, positive anticipated emotions did predict change in performance $\left[F(1,18)=7.21, p=.015, \eta^{2}=.30\right]$. However, there was no association between negative anticipated emotions and change in task performance. These findings partially support our second hypothesis. For the control condition, positive and 
negative anticipated emotions did not predict change in task performance condition, thus supporting our third hypothesis.

\section{Discussion}

This study was designed to examine the effects of counterfactual thinking on task performance during initial IT skills training. The current results indicate that generating counterfactual thoughts about one’s early task performance is, by itself, not a useful tool in facilitating improvement in a single-session introductory IT training context. Novice Excel users who were asked to generate counterfactual thoughts did not differ from learners who were not asked to think counterfactually in their improvement in performance in a second task.

Our secondary aim was to examine the relationship of anticipated emotions with goal focused performance in IT training, and how this relationship may be moderated by counterfactual thinking. A novel finding of our study was that a higher level of anticipated positive emotions was associated with greater improvement in task performance, but only for participants that were also asked to think counterfactually. Notably, even though negative anticipated emotions were not found to facilitate task performance, there was no evidence that such emotions impeded performance either. As shown in our follow-up analysis, this difference was not due to a difference in anticipated emotions between the counterfactual and control groups at the outset of the study. Our finding therefore supports our second hypothesis, and partially replicates the results of Leone et al. (2004) that positive anticipated emotions are associated with superior task performance when novices learn to use a computer software application.

Positive anticipated emotional consequences of success have been found in other studies to encourage success-facilitating behaviours in various domains (e.g., Dunton and Vaughan 2008, Leone et al. 2004). This study showed that anticipated positive emotions only 
benefit the task performance of participants in the counterfactual condition and negative anticipated emotions were not found to facilitate task performance.

While it may be tempting to attribute the difference in findings to task or sample characteristics, a more likely explanation is that the nature of the IT skills training in the present study was the primary contributor to the observed pattern of findings. Our interest was in how positive and negative emotions might relate to novice Excel users' initial training outcomes in learning to use the software. Similar to Leone et al.’s (2004) study, participation in the present study was voluntary, and participants' learning outcomes had no bearing on their academic studies. However, one further characteristic of the present study was that training entailed a single-session introduction to Excel and not an extensive training program. Hence participants might be primarily motivated by the positive gains that they could obtain from goal achievement, as opposed to anticipated emotions with regards to failing to achieve their goals. By comparison, in other studies that have found that negative anticipated emotions influenced behavioural intentions (e.g., Bagozzi and Dholakia 2006) or actual goal attainment (Leone et al. 2004), the cost of failing to achieve one's goals were higher than that in the present study. For example, having invested time and effort into participating in open source software user groups, group members were conceivably more motivated by the fear of failing to reap the benefits of participating as opposed to anticipation of positive gains (Bagozzi and Dholakia 2006). Likewise, having committed to spending a substantial amount of time in learning to use a software program, learners might again be more motivated by anticipated negative emotions arising from not adequately achieving their goals (Leone et al. 2004). The roles of learners' perceived gains versus losses at the outset of training may therefore be an important factor to consider in future research, which will benefit the design of learning environments that maximise learning outcomes. 
The theoretical connections among counterfactual thinking, behavioural intentions, and actual behaviour, as proposed by the functional theory of counterfactual thinking (Epstude and Roese 2008), may be useful in explaining why only positive anticipated emotions had a direct relationship with task performance in the counterfactual condition. According to this theory, counterfactual thinking typically entails producing causal statements that link achieving one's goal with factors or actions that are perceived to facilitate goal achievement. Such "what if” and "if only” thoughts in turn heighten behavioural intentions to engage in relevant actions or make good use of relevant factors. The end result is a greater likelihood of identifying and implementing success-facilitating behaviours when a similar situation arises (Epstude and Roese 2008).

Applying this account to the context of the present study, participants in both experimental conditions were primed to anticipate the emotional consequences of achieving or failing to achieve their learning goal in the training session. This would encourage all participants to set their personal reference standard of success. However, only those in the counterfactual condition were additionally prompted to generate counterfactual thoughts after the first experimental task ${ }^{6}$. Therefore they had the opportunity to identify relevant successfacilitating behaviours and factors, and would have heightened intentions to incorporate them in future. By comparison, participants in the control condition were not given this opportunity to search for success-facilitating behaviours and to formulate intentions to act accordingly. The experience of participants in the control condition should be further contrasted against that of participants in studies that assessed the MGB, which typically included assessing participants' intentions or desires to engage in a set of specified relevant success-facilitating behaviours (e.g., Bagozzi and Dholakia 2006; Leone et al. 2004; Perugini and Bagozzi 2001). In other words, in studies examining the MGB that demonstrated an influence of anticipated emotions, behaviours relevant to goal achievement were pre-defined for participants, thus 
bypassing the process of participants having to figure out those relevant behaviours themselves, such as what may be achieved via counterfactual thinking. Hence it is possible that what binds together anticipated emotions, behavioural intentions and corresponding behaviour may be the extent to which learners are aware of appropriate actions and factors for goal achievement. This possibility should be investigated further in future studies. This study represent one of the first applications of counterfactual thinking as an IT training strategy, and its impact on task performance. However, given the sample size of this initial investigation, further research with a larger sample is warranted. 


\subsection{Conclusions}

This is the first study to our knowledge where an intervention based on counterfactual thinking has been used to enhance task performance in an IT training context. Our findings have at least two practical implications for training novice learners to use IT applications. First, given the ease of incorporating instructions for learners to generate counterfactuals within the IT training context, it may be a cost-effective training strategy to include a prompt for learners to reflect on their initial task performance via generating counterfactual thoughts. Such goal-oriented reflections may prove beneficial in augmenting training outcomes when used in conjunction with other motivational strategies. Second, increasingly more people are undergoing training in IT skills to satisfy vocational as well as everyday needs. Our data suggest that an approach to creating an initial learning environment that facilitates task performance may involve two-steps : (i) Learners may be encouraged at the outset of training to anticipate the affective consequences of successfully achieving their learning goals versus failing to achieve such goals; this can be followed by (ii) prompts to encourage learners to generate counterfactual thoughts about their initial learning experience to motivate them to develop behavioural intentions for further improvement.

This study adds to the earlier body of knowledge regarding factors that may influence task performance when novice users are learning to acquire IT skills. We have shown early evidence for the relevance of counterfactual thinking and anticipated emotions in the IT training context. These are two areas that had been researched in other domains but relatively unexplored in the IT training context until now. We have identified a number of directions for future research; these pursuits may offer useful insight into how novice users may develop their competence in IT skills in a cost-effective way with satisfying practical and affective outcomes. 


\section{Footnotes}

1. We tested 58 participants in total (33 in the counterfactual condition, 25 in the control condition). However, data from 14 participants (11 participants from the counterfactual condition and 3 participants from the control condition) were incomplete and had to be excluded prior to data analysis. Two further participants (one from each of the counterfactual and control conditions) were tested but excluded from data analysis. Their solution procedures, as shown in their Camtasia recordings, clearly suggested that they had sufficient previous experience using Excel (one participant used pre-programmed formulae and the other used advanced formatting tools during the task). Of the 11 participants from the counterfactual condition whose data were incomplete and excluded, 6 were excluded because they failed to follow task instructions in the first experimental task (Task 1) and their data therefore could not be coded; 1 failed to follow instructions in the second experimental task (Task 2); 2 had missing data on Task 1; and 2 had missing data on Task 2. For the three participants from the control condition whose data were excluded, one was given a faulty spreadsheet for Task 1 due to technical error; one failed to follow instructions in Task 2 and his/her data therefore could not be coded; and the Camtasia file for one participant's Task 2 was not saved due to technical error and his/her data therefore could not be coded. Given that the majority of these exclusions were due to problems related to participants’ behaviour in Task 1 (i.e., before participants were presented with the counterfactual or control task), it was not the case that our experimental manipulations resulted in more participants from the counterfactual condition to be excluded. The final sample included 21 participants in each condition.

2. The purpose of including the second worksheet was to prevent individual differences in participants' ability to memorise the procedures learned in the training phase to affect their experimental task performance. 
3. Due to the exclusion of some participants (see Footnote 1), the number of participants allowed to sign up to latter testing sessions had to be varied to maintain the same number of participants in the counterfactual and control conditions in our final sample.

4. The second experimental task was unexpected because people are more likely to spontaneously generate upward counterfactuals if they expect to come across a similar event again (Markman et al. 1993). Inasmuch as the filler task for the control condition was set up to prevent spontaneous counterfactual thinking in participants from the control condition, we additionally refrained from informing participants from either condition earlier about the second experimental task. This was done as a further safeguard against counterfactual thoughts from occurring naturally in participants in the control condition.

5. A preliminary analysis showed that task order did not affect participants’ performance scores.

6. It may be argued that the opportunity for participants in the counterfactual condition to reflect on their initial experimental task performance (via writing a description of their task performance) was instrumental to their subsequent greater improvement in task performance. Note, however, that in prior research which included a "reflection only" control condition, it has been demonstrated that greater intentions to engage in successfacilitating behaviour in future do not arise if one simply reflects on a performance-oriented experience without also thinking counterfactually about it (Roese 1994, Experiment 2). 


\section{Acknowledgement}

The authors wish to thank Danielle Stockwell for assisting with data collection for the present study. 


\section{Appendix A}

Instructions for experimental tasks

(with number of answers required for each step appearing in square brackets)

\section{Property Task}

John is a property investor. He has created an Excel sheet to calculate the earnings from buying and selling his three investment properties. Using the Excel skills you have learned today, please help John complete his calculations by carrying out the following steps:

1. Calculate No. of Years Held for the Suburb A property by subtracting year purchased from year sold. [1]

2. Calculate Overall Profit for Suburb A property by subtracting the purchase price from the sale price. [1]

3. Calculate Profit After Expenses for Suburb A property: This is the overall profit minus costs. [1]

4. Calculate \% Gain (Overall): This equals Profit After Expenses divided by Purchase Price. [1]

5. Calculate \% Gain Per Year: This is \% Gain (Overall) divided by the number of years held. [1]

6. Use an efficient method to carry out all of the above calculations for the Suburb B and Suburb C properties. [10: 1 answer x 2 suburbs x 5 questions (i.e., Questions 1-5)]

7. Calculate Total Overall Profit: this is the sum of the overall profit for the 3 properties. [1]

8. Calculate Total Profit After Expenses, i.e., sum of the 3 Profit After Expenses figures. [1]

9. Calculate the Average Profit After Expenses for the 3 properties. [1]

10. Calculate the Average Percentage Gain Per Year. [1] 


\section{Chocolate Task}

Jane sells hand-made chocolates at Easter. She has created an Excel sheet to calculate her earnings this year from this business. Using the Excel skills you have learned today, please help Jane complete her calculations by carrying out the following steps:

1. Calculate Production Cost for Decorated Easter Eggs by multiplying Production Cost Per Unit by Quantity Produced. [1]

2. Calculate Overall Profit for Decorated Easter Eggs by multiplying the per unit retail price by the quantity sold. [1]

3. Calculate Profit After Expenses for Decorated Easter Eggs: This equals Overall Profit minus Production Cost. [1]

4. Calculate Total Production Time: This is the Quantity Produced multiplied by Production Time Per Unit. [1]

5. Calculate Profit Per Hour: This is the Profit After Expenses divided by the Total Production Time. [1]

6. Use an efficient method to carry out all of these calculations for the Plain Easter Eggs and Chocolate Bunnies. [10: 1 answer x 2 suburbs x 5 questions (i.e., Questions 1-5)]

7. Calculate Total Overall Profit: this is the sum of the Overall Profit for the 3 products. [1]

8. Calculate Total Profit After Expenses, i.e., sum of the 3 Profit After Expenses figures. [1]

9. Calculate the Average Profit After Expenses for the 3 products. [1]

10. Calculate the Average Profit Per Hour. [1] 


\section{Appendix B}

Additional examples of counterfactual thoughts generated by participants Upward counterfactuals

If only I had checked the formulas I put in for typing errors more than once or twice. (Participant 3797WI0986)

If only I'd been able to keep calm when I experienced the problem. (Participant 4545BA1286)

If only I remembered how to correctly work out averages. (Participant 7301MC0186)

What if someone was able to help me while I was doing it. (Participant 1983MC1186)

If I had more time, I may have been able to finish the task satisfactorily. (Participant 2245KO0187)

Downward counterfactuals

What if I typed each individual cell formula. I wonder how much longer that would have taken. (Participant 3808LE0387)

What if the method I tried was wrong... (Participant 6461PA0286)

If I hadn't been shown how to perform various functions on Excel, I still feel I could have completed the task, but MUCH less efficiently. Probably cell by cell and without copy and paste - a lot longer. (Participant 9726BR1086)

What if there were a shortened time limit to complete the task. (Participant 3808LE0387) 


\section{References}

Bagozzi, R.P., Dholakia, U.M., 2006. Open source software user communities: A study of participation in Linux user groups. Management Science, 52 (7), 1099-1115.

Carver, C. S., Scheier, M. F., 1998. On the self-regulation of behavior. Cambridge, UK: Cambridge University Press.

Debowski, S., Wood, R.E., Bandura, A. 2001. Impact of guided exploration and enactive exploration on self-regulatory mechanisms and information acquisition through electronic search. Journal of Applied Psychology, 86 (6), 1129-1141.

Dunton, G.F., Vaughan, E. 2008. Anticipated affective consequences of physical activity adoption and maintenance. Health Psychology, 27 (6), 703-710.

Epstude, K., Roese, N.J., 2008. The functional theory of counterfactual thinking. Personality and Social Psychology Review, 12(2), 168-192.

Galinsky, A.D., Kray, L.J., 2004. From thinking about what might have been to sharing what we know: The effects of counterfactual mind-sets on information sharing in groups. Journal of Experimental Social Psychology, 40, 606-618.

Galinsky, A.D., Moskovitz, G.B., 2000. Counterfactuals as behavioural primes: Priming the simulation heuristic and consideration of alternatives. Journal of Experimental Social Psychology, 36, 384-409.

Gist, M.E., Schwoerer, C.E., Rosen, B., 1989. Effectes of alternative training methods on Self efficacy and performance in computer software training. Journal of Applied Psychology 74(6), 884-891.

Gleicher, F., Boninger, D.S., Strathman, A., Armor, D., Hetts, J., Ahn, M., 1995. With an eye toward the future: The impact of counterfactual thinking on affect, attitudes, and behaviour. In: Roese, N.J., Olson, J.M. eds., What might have been: The social 
psychology of counterfactual thinking. Mahwah, NJ: Lawrence Erlbaum Associates, 283-304.

Harris, P., German, T., Mills, P., 1996. Children’s use of counterfactual thinking in cause reasoning. Cognition, 61, 233-259.

Keith, N., Frese, M., 2005. Self-regulation in error management training: Emotion control and metacognition as mediators of performance effects. Journal of Applied Psychology, 90, 677-691.

Kray, L.J., Galinsky, A.D., 2003. The debiasing effect of counterfactual mind-sets on group decisions: Increasing the search for disconfirmatory information in group decisions. Organizational Behavior and Human Decision Processes, 91, 69-81.

Kray, L.J., Galinsky, A.D., Wong, E.M., 2006. Thinking within the box: The relational processing style elicited by counterfactual mind-sets. Journal of Personality and Social Psychology, 91, 33-48.

Leone, L., Perugini, M., Ercolani, A.P. 2004. Studying, practicing, and mastering: A test of the model of goal-directed behavior (MGB) in the software learning domain. Journal of Applied Social Psychology, 34 (9), 1945-1973.

Mandel, D. R., Lehman, D. R. 1996. Counterfactual thinking and ascription of cause and preventability. Journal of Personality and Social Psychology, 70, 450-463.

Markman, K.D., Gavanski, I., Sherman, S.J., McMullen, M.N., 1993. The mental simulation of better and worse possible worlds. Journal of Experimental Social Psychology, 29, 87-109.

Markman, K.D., Lindberg, M.J., Kray, L.J., Galinsky, A.D., 2007. Implications of counterfactual structure for creative generation and analytical problem solving. Personality and Social Psychology Bulletin, 33 (3), 312-324. 
Page, C. M., Colby, P. M., 2003. If only I hadn't smoked: the impact of counterfactual thinking on a smoking-related behavior. Psychology and Marketing, 20, 955-976.

Perugini, M., Bagozzi, R.P. 2001. The role of desires and anticipated emotions in goaldirected behaviours: Broadening and deepening and theory of planned behaviour. British Journal of Social Psychology, 40, 79-98.

Roese, N.J., 1994. The functional basis of counterfactual thinking. Journal of Personality and Social Psychology, 66, 805-818.

Roese, N. J., Olson, J. M., 1993. The structure of counterfactual thought. Personality and Social Psychology Bulletin, 19, 312-319.

Roese, N.J., Olson, J.M., 1995. Functions of counterfactual thinking. In: N.J. Roese and J.M. Olson eds., What might have been: The social psychology of counterfactual thinking. Mahwah, NJ: Lawrence Erlbaum Associates, 169-197.

Torrance, E.P., Ball, O.E., Safter, H.T., 1990. Torrance Tests of Creative Thinking. Scholastic Testing Service, Bensenville, IL.

Tsiro, M., Mittal, V., 2000. Regret: A model of its antecedents and consequences in consumer decision making. Journal of Consumer Research, 26 (4), 401-417.

van der Pligt, J., de Vries, N. K., 1998. Expectancy-value model of health behaviors: The role of salience and anticipated affect. Psychology and Health, 13, 289-305.

Yi, M.Y., Davis, F.D., 2003. Developing and validating an observational learning model of computer software training and skill acquisition. Information Systems Research, 14(2), 146-169. 


\section{Figure Captions}

Figure 1. Initial setup of the Property practice task.

Figure 2. Mean experimental task performance scores by experimental condition 


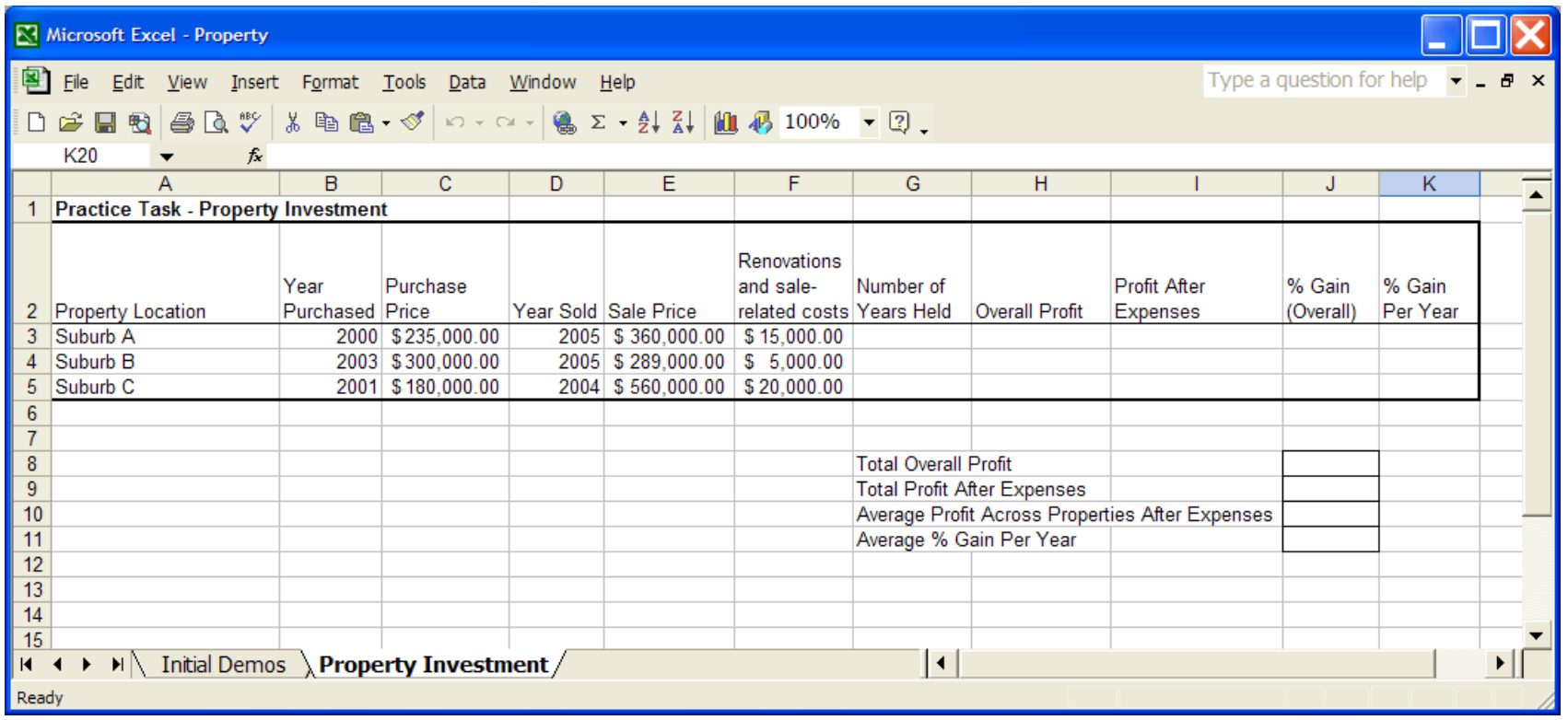




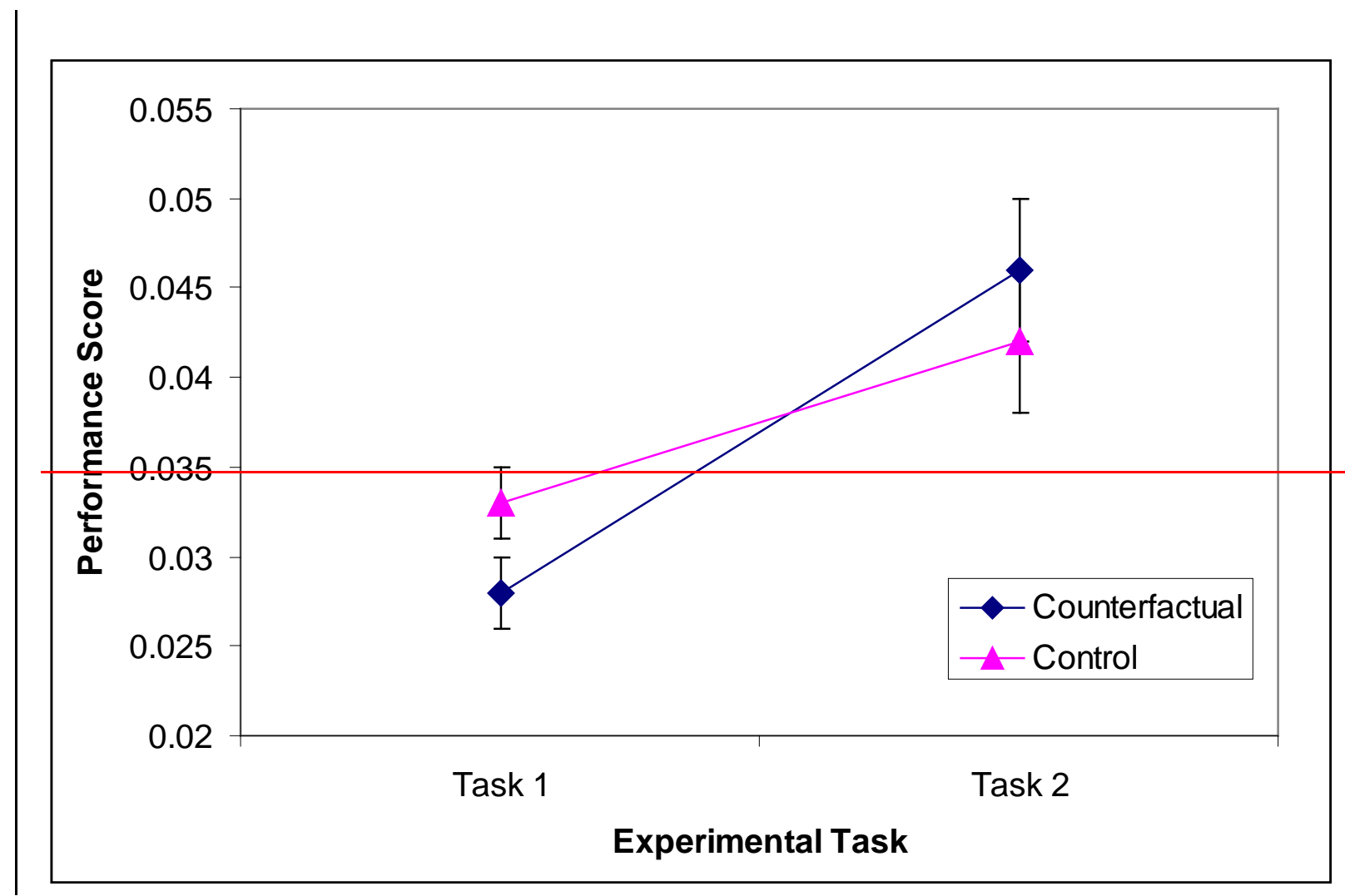

